\title{
Kulturelle Bildung
}

\section{Annette Scheunpflug • Christoph Wulf · Ivo Züchner}

Angenommen: 17. Dezember 2020 / Online publiziert: 7. Januar 2021

(C) Der/die Autor(en) 2021

2013 hat sich die Zeitschrift für Erziehungswissenschaft in ihrem Sonderheft 21 zum letzten Mal dezidiert mit der „Kulturellen Bildung“ beschäftigt (Scheunpflug und Prenzel 2013). Ein Blick in dieses Heft macht deutlich, wie sehr es vor sieben Jahren noch um die Vermessung des Terrains ging und die Frage, wie sich die Situation der kulturellen Bildung überhaupt beschreiben ließe, im Mittelpunkt des Interesses stand. Damals wurde die Diskussion um die kulturelle Bildung im Modus der Sorge geführt, stand sie doch seit dem Wissen um die Leistungsmängel des deutschen Schulwesens nach PISA zumindest subjektiv unter Druck. Parallel mit dem Bildungsbericht von 2012 zum Schwerpunktthema „kulturelle Bildung“ (Arbeitsgruppe Bildungsberichterstattung 2012; Grigic und Rauschenbach 2013), ging es darum, den Stellenwert der kulturellen Bildung in verschiedenen Bildungsbereichen zu erfassen, Transfereffekte kultureller Bildung zu beschreiben und für die Bedeutung dieses Forschungsfeldes zu sensibilisieren. Auch international ist diese Form der legitimierenden und das Feld beschreibende Forschung inzwischen gut etabliert (vgl. z.B. Ijdens 2016).

Prof. Dr. A. Scheunpflug $(\bowtie)$

Lehrstuhl für Allgemeine Pädagogik, Otto-Friedrich-Universität Bamberg,

Markusplatz 3, 96047 Bamberg, Deutschland

E-Mail: annette.scheunpflug@uni-bamberg.de

Prof. Dr. C. Wulf

Arbeitsbereich Anthropologie und Erziehung, Freie Universität Berlin,

Habelschwerdter Allee 45, 14195 Berlin, Deutschland

E-Mail: christoph.wulf@fu-berlin.de

Prof. Dr. I. Züchner

Institut für Erziehungswissenschaft, Philipps Universität Marburg,

Wilhelm-Röpke-Straße 6, 35032 Marburg, Deutschland

E-Mail: zuechner@staff.uni-marburg.de 
Inzwischen hat sich dieses Thema deutlich weiterentwickelt. Die Bedeutung kultureller Bildung ist in das gesellschaftliche wie wissenschaftliche Bewusstsein gerückt. Die gestiegene gesellschaftliche Bedeutung zeigt sich beispielsweise in der Profilierung von Kulturschulen, in der Etablierung von Förderprogrammen wie etwa „Kultur macht stark“ oder in den Aktivitäten des Rats für kulturelle Bildung (vgl. Rat für Kulturelle Bildung 2016, 2019). Der wissenschaftliche Bedeutungszuwachs wird in der Intensivierung der Forschungsaktivitäten zu diesem Thema erkennbar. Dazu hat sicherlich auch die Förderlinie des BMBF zur ,Forschung zur kulturellen Bildung“ beigetragen (vgl. BMBF 2020). Die Forschungslandschaft hat sich inzwischen erheblich ausdifferenziert (vgl. im Überblick Liebau et al. 2014; Timm et al. 2020). Neue Themen sind hinzugekommen, etwa die Digitalisierung, unterschiedliche Zielgruppen kultureller Bildung oder das informelle kulturelle Lernen. Es wird sowohl die Produktion wie die Reproduktion des Kulturellen in den Blick genommen. Methodisch hat sich das Feld weiterentwickelt - für die kulturelle Bildung wird inzwischen die breite Palette verschiedener Forschungsparadigmen genutzt. Theoretisch hat sich das Feld ausdifferenziert; die Forschung knüpft inzwischen sowohl an Fragen ästhetischer Bildung, an einen hochkulturellen Kulturbegriff oder gleichermaßen an einen bedeutungsorientierten Kulturbegriff im Sinne Clifford Geertz (2010 [1973]) an.

Mit diesem Thementeil möchten wir exemplarisch einen Blick in die Weiterentwicklung des Forschungsfeldes durch neuere empirische Studien werfen. Weitere interessante Studien werden wir in dem Anfang 2021 erscheinenden Band der Edition ZfE ebenfalls zum Thema „Kulturelle Bildung“ vorstellen.

Der Stichwortbeitrag von Stephan Kröner, Alexander Christ und Marcus Penthin gibt einen Überblick über die Forschung zur Digitalisierung in der kulturell-ästhetischen Bildung. Die Autorengruppe zeigt einen neuen, die Möglichkeiten digitaler Technik nutzenden Zugang zum Forschungsfeld auf und demonstrieren damit auch das Potenzial, das in entsprechenden Zugängen steckt. Auch wenn es sich im Hinblick auf die Digitalisierung in der kulturell-ästhetischen Bildung noch um ein sich entwickelndes Forschungsfeld handelt, wird deutlich, wie fruchtbar eine konfigurierende Forschungssynthese zur Kartierung dieses sich entwickelnden Forschungsfeldes sein kann. Die Autorengruppe identifizierte im Fachportal Pädagogik und in Scopus empirisch ausgerichtete Originalarbeiten mit explizitem Bezug zur Digitalisierung in der kulturellen Bildung. Die Beiträge wurden regelgeleitet kategorisiert. Inhaltlich wird ein Schwerpunkt in den bildenden Künsten deutlich. Sichtbar wird ein Forschungsdesiderat zu Entwicklungen in den Bereichen Robotik und der Virtual Reality sowie hinsichtlich non-formaler Bildungsprozesse. Einige methodische Schwächen des Forschungsfeldes, wie schwache Designs, naive Vergleiche zwischen Analogem und Digitalem oder unterausgewertete Datensätze werden identifiziert, um Empfehlungen für künftige Studien zu geben.

Die drei folgenden Beiträge setzen jeweils alle im Feld der Lehrerbildungsforschung an und adressieren unterschiedliche Facetten der kulturellen Bildung in diesem Feld.

Für die Ausbildung von Lehrkräften im Bereich kultureller Bildung spielen entsprechende Lehrbücher eine wichtige Rolle. Claudia Kühn, Konstantin Lindner und Annette Scheunpflug berichten über die Ergebnisse einer empirischen Diskursana- 
lyse. Sie haben fachdidaktische Lehrbücher der Englisch-, Geschichts- und Musikdidaktik untersucht und das diesen Lehrwerken zugrundeliegende Kulturverständnis herausgearbeitet. Mit der Studie wird deutlich, dass bedeutungsorientierte kulturtheoretische Konzepte zwar durchaus genutzt werden, um die Vielfalt kultureller Ausdrucksformen sichtbar zu machen, dass diese jedoch, wenn es um die schulische Praxis geht, dominant auf essentialisierende Kulturkonzepte zurückgreifen. Damit werden die theoretischen Anforderungen des „cultural turns“ für die Arbeit in der Schule zwar benannt, aber inhaltlich nicht durchgearbeitet. Das untersuchte Material bietet Lehrkräften zudem nur wenige Anregungen zu Selbstreflexion und zu metatheoretischen Deutungen des Kulturellen.

Der dritte Beitrag knüpft hier an, indem die Einstellungen von Lehramtsstudierenden in den Blick genommen werden. Susanne Timm blickt auf zukünftige Lehrkräfte als solche, die durch Kultur geprägt sind und zugleich in ihrer Profession als Kultur prägend tätig sind. Welche Orientierungen sind für diesen Personenkreis handlungsleitend? Auf der Basis von Gruppendiskussionen wurden in einer qualitativen Auswertung entsprechende Typen identifiziert. Dabei zeigte sich, dass über informelle Lernprozesse die Deutungen der Mehrheitskultur unreflektiert übernommen werden. Offensichtlich kann das universitäre Lehrprogramm nicht hinreichend Impulse erzeugen, um Orientierungen und Haltungen so zu modifizieren, dass zumindest die eigene Sozialisation kritisch reflektiert wird. Dies ist für die Verfasserin der Ausgangspunkt, um Empfehlungen und Anregungen für die Ausbildung von Lehramtsstudierenden zu formulieren.

Mit dem vierten Artikel dieses Thementeils, verfasst von Caroline Rau, werden die Orientierungen von Lehrkräften untersucht, die ein geisteswissenschaftliches Fach unterrichten. Welche handlungsleitenden Orientierungen zeigen Lehrkräfte hinsichtlich der erkenntnistheoretischen Grundlagen ihrer Fächer? Wie verstehen sie die Genese von Wissen in ihrem Fach und welche Formen der Konstruktion des Kulturellen werden darin erkennbar? Die Daten wurden auch hier in Gruppendiskussionen erhoben und nach der Dokumentarischen Methode ausgewertet. Die Verfasserin rekonstruiert unter dem Aspekt, wie die Lehrkräfte ihren Unterrichtsinhalten „Geltung“ verschaffen wollen, drei Idealtypen. Dieser Befund wird in verschiedenen Dimensionen diskutiert und im Hinblick auf die Rolle und den Umgang von kultureller Vielfalt und Kontingenz im Unterrichtsgeschehen ausgewertet.

Funding Open Access funding enabled and organized by Projekt DEAL.

Open Access Dieser Artikel wird unter der Creative Commons Namensnennung 4.0 International Lizenz veröffentlicht, welche die Nutzung, Vervielfältigung, Bearbeitung, Verbreitung und Wiedergabe in jeglichem Medium und Format erlaubt, sofern Sie den/die ursprünglichen Autor(en) und die Quelle ordnungsgemäß nennen, einen Link zur Creative Commons Lizenz beifügen und angeben, ob Änderungen vorgenommen wurden.

Die in diesem Artikel enthaltenen Bilder und sonstiges Drittmaterial unterliegen ebenfalls der genannten Creative Commons Lizenz, sofern sich aus der Abbildungslegende nichts anderes ergibt. Sofern das betreffende Material nicht unter der genannten Creative Commons Lizenz steht und die betreffende Handlung nicht nach gesetzlichen Vorschriften erlaubt ist, ist für die oben aufgeführten Weiterverwendungen des Materials die Einwilligung des jeweiligen Rechteinhabers einzuholen. 
Weitere Details zur Lizenz entnehmen Sie bitte der Lizenzinformation auf http://creativecommons.org/ licenses/by/4.0/deed.de.

\section{Literatur}

Arbeitsgruppe Bildungsberichterstattung (2012). Bildung in Deutschland 2012. Ein indikatorengestützter Bericht mit einer Analyse zur kulturellen Bildung im Lebenslauf (gefördert mit Mitteln der Ständigen Konferenz der Kultusminister der Länder in der Bundesrepublik Deutschland und des Bundesministeriums für Bildung und Forschung, unter Federführung des DIPF). Bielefeld: wbv.

Bundesministerium für Bildung und Forschung (BMBF) (2020). Kulturelle Bildung. https://www.bmbf. de/de/kulturelle-bildung-5890.html. Zugegriffen: 10. Juli 2020.

Geertz, C. (2010). Dichte Beschreibungen. Beiträge zum Verstehen kultureller Systeme. Frankfurt a. M.: Suhrkamp. (2010/1973)

Grgic, M., \& Rauschenbach, T. (2013). Kulturelle Bildung im Horizont der Bildungsberichterstattung des Bundes (2012/13). https://www.kubi-online.de/artikel/kulturelle-bildung-horizont-bildungsbericht erstattung-des-bundes. Zugegriffen: 06. Jan. 2021.

Ijdens, T. (2016). Monitoring National Arts Education Systems (MONAES): some results of two surveys among arts education experts around the world. Utrecht: Netherlands Centre of Expertise for Cultural Education and Amateur Arts.

Liebau, E., Jörissen, B., \& Klepacki, L. (Hrsg.). (2014). Forschung zur Kulturellen Bildung. Grundlagenreflexionen und empirische Befunde. München: kopaed.

Rat für kulturelle Bildung (2016). Studie: Städte/Geld/Kulturelle Bildung. Essen: Rat für Kulturelle Bildung e.V.

Rat für kulturelle Bildung (2019). Studie „Jugend/YouTube/Kulturelle Bildung“. Essen: Rat für Kulturelle Bildung e.V.

Scheunpflug, A., \& Prenzel, M. (Hrsg.). (2013). Kulturelle und ästhetische Bildung (Zeitschrift für Erziehungswissenschaft: Sonderheft 21). Wiesbaden: Springer VS.

Timm, S., Costa, J., Kühn, C., \& Scheunpflug, A. (Hrsg.). (2020). Kulturelle Bildung. Theoretische Perspektiven, methodologische Herausforderungen und empirische Befunde. Münster: Waxmann. 